\title{
IMAGING STELLAR CORONAE FROM ECLIPSING BINARY
} X-RAY LIGHT CURVES

\author{
M. SIARKOWSKI \\ Space Research Center, Polish Academy of Sciences \\ Kopernika 11, 51-622 Wroctaw, Poland
}

\section{Introduction}

The Sun is the only star whose X-ray emitting, strongly inhomogenous corona can be spatially resolved via direct observations. For other late typestars it is known that coronae do exist, but the spatial distribution of their emission is largely unknown. However in the case of eclipsing binaries this spatial structure can be potentially deduced from the orbital modulation of the observed X-ray light curve. The best candidates for this kind of analysis are RS CVn binaries, the most active and luminous late-type $\mathrm{X}$-ray coronal sources. These are detached binaries with periods typically between 0.5 and 20 days, in which one or both stars have evolved into subgiant or giant of spectral type G or K. For short orbital periods ( $<14$ days) the tidal forces lead to synchronization of the orbital and rotational periods, so these systems rotate rigidly.

The application of eclipse imaging to stellar coronae was pioneered by Walter, Gibson, and Basri (1983), using Einstein IPC and MPC observations of AR Lac eclipsing binary, made at various orbital phases, covering $17 \%$ of an orbital cycle. The authors modeled the corona of AR Lac as a system of shells or toruses with emission flux modulated sinusoidally in longitude. Modeling the light curve around eclipses, they found that both stars appear to have compact coronal structures with scale heights small $\left(\sim 0.02 \mathrm{R}_{\star}\right)$ compared to the stellar radius. In addition, a highly inhomogenous, extended X-ray corona with scale height of about $1 R_{\star}$ appears to be associated with the secondary $\mathrm{K}$ star.

Another imaging technique has been used by White et al. (1990) for the analysis of the EXOSAT observations of AR Lac, which completely covered one orbital period. The authors used $\chi^{2}$ fitting of the observed light curve 
in order to identify a number of uniformly emitting structures, and the maximum entropy method, which directly maps the emission distribution on stellar surfaces. Each individual structure was described by uniformly emitting, truncated spherical cone with its apex at the stars center. Such structure was characterised by its position, a cone half-angle, a height above stellar surface, and intensity. To account for any unmodulated emission a constant component of emission was included as free parameter. The same $\chi^{2}$ fitting technique was used by Culhane et al. (1990) for analysis of the EXOSAT observations of the eclipsing RS CVn binary TY Pyx, and by Ottmann et al. (1993) for the ROSAT observations of AR Lac (this later also with pyramids instead of conical structures).

Some other, rather qualitative techniques were used for analysis of the coronal structure in the RS CVn system XY UMa (Bedford et al. 1990) and active solar-type dwarf V471 Tauri (Guinan and Carroll 1990).

In this contribution, I present the results of imaging of stellar coronae as obtained using the three-dimensional imaging technique (Siarkowski 1992) for AR Lac EXOSAT, ROSAT and ASCA data and for TY Pyx EXOSAT observations. In this method we divide the volume around the binary system into a grid of elementary cubes (pixels) and change their emissivity iteratively until reconstruction of the observed X-ray light curve to within the statistical errors. As in the previous methods we assume that the system rotates as rigid body, and that the emission is optically thin and stable at least over a time-scale of one orbital period. However in our approach we do not need to make any assumption about the localisation, number or size of the emitting regions. We assume symmetry relative to the orbital plane $\left(\mathrm{i}=90^{\circ}\right)$.

\section{Exosat}

\subsection{AR LAC}

AR Lac is an eclipsing RS CVn binary system with a 1.983 day period and orbital inclination $i=87^{\circ}$. It contains a G2IV primary (with radius $\mathrm{R}$ $\left.=1.54 \mathrm{R}_{\odot}\right)$ and a K0IV secondary $\left(\mathrm{R}=2.81 \mathrm{R}_{\odot}\right)$ separated by $9.22 \mathrm{R}_{\odot}$, and having approximately equal masses of $1.35 M_{\odot}$ (Chambliss 1976). As a relatively nearby $(\sim 50 \mathrm{pc})$ system, it is one of the brightest stellar coronal $\mathrm{X}$-ray source with $\mathrm{X}$-ray luminosity, $\mathrm{L}_{x} \simeq 10^{31} \mathrm{ergs} \mathrm{s}^{-1}$.

AR Lac was observed by EXOSAT in two energy bands in July 1984 for a one full orbital cycle with almost continuous phase coverage (White et al. 1990). The low energy, LE, 0.05-2 keV light curve showed a clear orbital modulation (with a $50 \%$ reduction in flux during the primary eclipse). In contrast, no orbital modulation was detected at the medium energy, ME, 1-6 keV light curve, where emission with temperatures above $10 \mathrm{MK}$ dom- 
inates. The X-ray spectra of the RS CVn type stars measured with the Einstein SSS and EXOSAT TGS show at least two temperature components to be present at 5-7 and 15-40 MK (Swank et al. 1981, Lemen et al. 1989). The deep modulation in the LE light curve suggests compact lowertemperature structures to be present close to the surface of one or both of the stars. The ME light curve is dominated by the hotter component and the lack of any strong modulation suggested this component comes from a large region comparable in size to the binary system dimensions.

By fitting the LE light curve ( $\chi^{2}$ optimization), White et al. (1990) found five different solutions which reproduce the observations. In each of the solution two or three localized, compact structures of emission on one or both stars were found in addition to a constant uneclipsed extended region. In some model solutions, a second more extended structure with a height of 1 to $4 R_{\odot}$ was necessary to fit the observations.

The same LE light curve has been used by Siarkowski (1992) to test the $3 \mathrm{D}$ imaging technique. He found that both stars have active coronae (see Fig. 2a) with many emitting structures, with the largest having sizes greater than the stellar radii. The main features are large complexes of activity located on each star and an extended, more diffuse structure anchored to the surface of the K star. The solution presented in Fig. 2a was obtained iteratively starting from uniform emission distribution within the system, (very similar image appears when starting from the "negative" of the resulting image). The solution obtained is also stable against small perturbations of the binary system parameters. It is also important that nearly all individual coronal structures found by White $e t$ al. (1990) are present in Fig. 2a, so it can be stated that 3D imaging method combines all discrete solutions obtained by White et al. into one.

\subsection{TY PYX}

TY Pyx (HD 77137) is a 3.2 day eclipsing $\left(i=88^{\circ}\right)$ RS CVn binary system. It consists of two similar solar-like stars (G2-5 V) with radii $\mathrm{R}=1.59 \mathrm{R}_{\odot}$ and $\mathrm{R}=1.68 \mathrm{R}_{\odot}$, respectively, separated by $12.25 \mathrm{R}_{\odot}$. This system was observed by EXOSAT low-energy imaging telescope, LEIT (0.05-2 keV), and medium energy proportional counter, ME (1-30 keV), during one complete orbital binary period. The obtained soft X-ray (LE) light curve is clearly modulated due to eclipses in the binary system. As in the EXOSAT observations of AR Lac, no orbital modulation was detected at the medium energy (ME).

The LE light curve modulation has been modeled by Culhane et al. (1990) using $\chi^{2}$ fitting technique similar to that used for AR Lac by White et al. (1990). Their results show that the observed X-ray light curve can 
result from eclipsing of two emission regions, opposing each other on the inward faces of the two stars (i.e. having longitudes $\sim 0^{\circ}$ and $180^{\circ}$ ). The authors assumed that the vertical size of emitting structures is $0.01 R_{\odot}$, similar to the size found in the earlier modelling of AR Lac system, however the possibility that the two regions form one continuous region between the two stars had not been excluded.

The LE light curve was reanalysed by Siarkowski et al. (1994) and by Preś et al. (1995a) using a 3D imaging technique. The results obtained (Fig. 1) indicate for the presence of the bulk of plasma in between the two stars. The spatial distribution of the emission indicates for the presence of the loop-like structure interconnecting the two stars. This loop-like structure is responsible for nearly $40 \%$ of the total emission while the active regions identified on the surface of both stars contribute to the residual flux observed during eclipses. These structures constitute also an important contribution to the flux outside the eclipses. The contribution of the residual emission pervading whole binary system (halo emission) is found to be less than $2 \%$.

There are three kinds of emission models which can explain observed LE light curve modulation: two compact regions on the inward faces of the two stars, elongated structure between both stars and two elongated structures at the outward faces of both stars. Extensive test simulations performed by Preś et al. (1995a) indicate however, that 3D deconvolution technique can in principle distinguish between these solutions.

However, the uncertainties in the observed light curve are so large, that even with the emission assumed to be uniform within the system, the obtained value of a reduced $\chi^{2}$ is as low as 1.75. In this context Culhane's solution cannot be definitely rejected, but comparison of the observed and calculated light curves (Fig. 1) indicates that interconnecting loop model seems to explain the observed modulation more accurately.

Preś et al. (1995a) have also modeled physical conditions in this loop using the hydrostatic model of the coronal loop with varying cross-section area (Vesecky et al. 1979). The brightness distribution along the observed interconnecting structure can be interpreted assuming hydrostatic loop models with $12 \mathrm{MK}<\mathrm{T}_{\max }<48 \mathrm{MK}$ and $\Gamma$ (the ratio of the largest to smallest loop cross-section areas) $>15$. The plasma density for the acceptable models are in the ranges from $210^{8} \mathrm{~cm}^{-3}$ to $310^{10} \mathrm{~cm}^{-3}$.

\section{ROSAT}

AR Lac was extensively observed with ROSAT PSPC $(0.1-2.4 \mathrm{keV})$ : during in-orbit calibration phase in July 1990, and in all-sky survey phase in December 1990. 

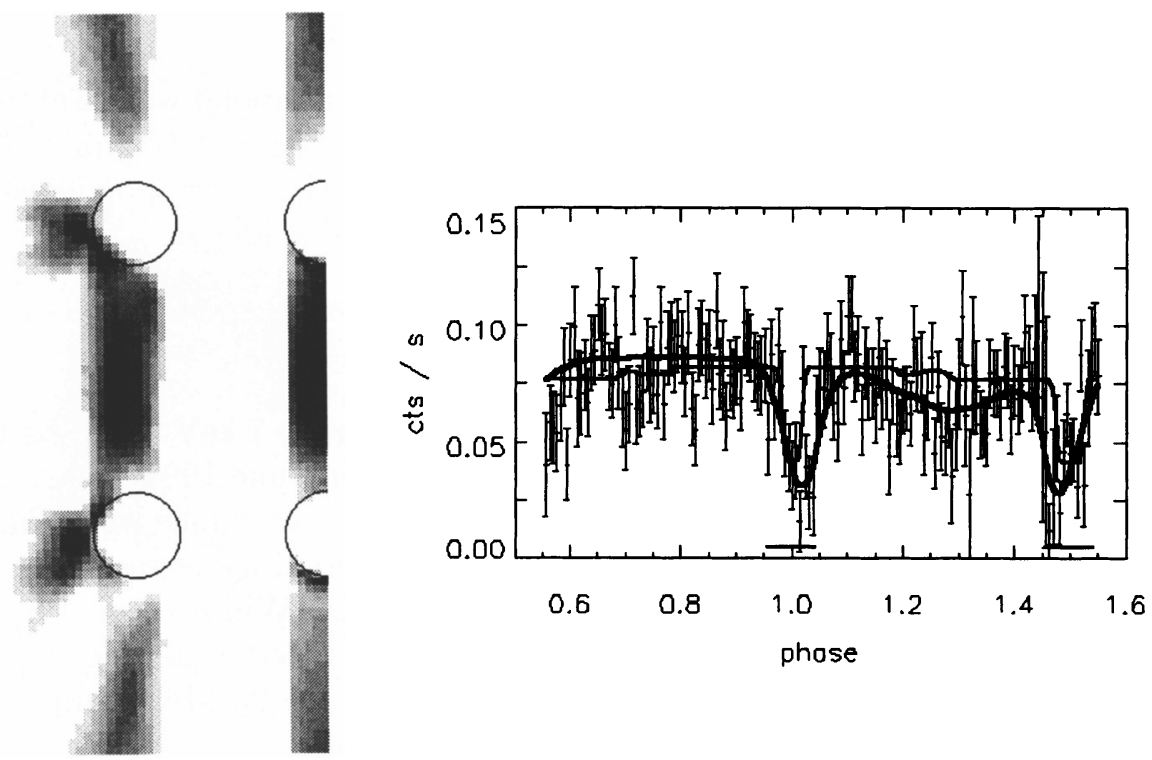

Figure 1. A model for (LE) $0.05-2 \mathrm{keV}$ X-ray coronal emission in the TY Pyx binary (Preś et al. 1995a). From the left: the top view, the side view and comparison of the observed light curve with these calculated for loop model (thick line) and the model of Culhane et al. (1990, thin line).

Observation obtained during calibration phase was analysed by Ottmann et al. (1993). In contrast to the EXOSAT results these observations showed the primary eclipse at all energies. Using $\chi^{2}$ fitting technique to modeling the total light curve, the authors found three different solutions. All models obtained require one prominent coronal feature on each star, a compact structure on the $\mathrm{G}$ star (size $\left(\sim 0.03 \mathrm{R}_{\odot}\right)$ and more extended structure associated with the $\mathrm{K}$ star (size $\sim 1-2 \mathrm{R}_{\odot}$ ).

The PSPC total light curve obtained during ROSAT all-sky survey (as presented by Schmitt, 1992) was analyzed by Preś et al. (1995b). This observation covers more than one orbital cycle. Toward the end of the observation period major flaring activity occurred. Fortunately, prior to this flare event, nearly one full binary cycle (from phase 0.65 to 1.56) of quiescent emission was recorded with primary eclipse clearly present.

$3 \mathrm{D}$ deconvolution of this light curve (Fig. $2 \mathrm{~b}$ ) leads to the image of the corona with a few strong compact structures located near the surfaces of both stars and more or less uniform halo emission pervading the whole binary. Also a small compact structures located high $\left(\sim 1-2 R_{\star}\right)$ above stellar surfaces are present in this solution. Contribution of all these structures to the total emission is important. The halo, active regions near stellar surfaces and other compact structures constitutes $44 \%, 33 \%$ and $23 \%$ 
of emission respectively.

Location of two largest regions on the $\mathrm{K}$ star correspond well to extended structures from models of Ottmann et al. (1993). Since Ottmann's models describe the status of corona half a year earlier, this correspondence may indicate that life-time of active regions in AR Lac is longer.

\section{ASCA}

The X-ray light curves in the $0.4-1.5 \mathrm{keV}$ (SIS) and 2-7 keV (GIS) bands of AR Lac were observed over one full orbital cycle in June 1993 (White et al. 1994). In contrary to EXOSAT results but in full accordance with Einstein and ROSAT data, deep primary and shallow secondary eclipses were observed in both energy channels. Spectral analysis (White et al. 1994 and Singh et al. 1995) indicates that emission can be fitted assuming two temperature model with low $(\sim 7 \mathrm{MK})$ and the high $(\sim 23 \mathrm{MK})$ temperature components.

3D modeling of SIS and GIS light curves (Siarkowski et al. 1995) indicates (Fig 2c and d) that (i) both stars are X-ray active, (ii) the emission tends to be concentrated on the inward sides of the stars, (iii) there are compact, well localized regions of enhanced X-ray emission with heights well below one stellar radius, (iv) there is a 'halo' emission that pervades the entire system, and (v) there is indication for presence of emission region that links the two stars. There are also acceptable models in which only low lying sources are present without extended linked structures and halo emission. However, in this case only intensities of these low lying sources change preserving global sources distribution.

Observation of AR Lac in two fairly well-separated energy bands has provided an opportunity to estimate the physical parameters of the individual structures of coronae in this binary system. The electron densities inferred for the spatially resolved emission regions are similar to the range of values observed in the solar active regions $\left(10^{9}-10^{10} \mathrm{~cm}^{-3}\right)$, while the estimated temperatures correspond to the solar flares $(\sim 20 \mathrm{MK})$. In all considered solutions the temperature of sources around the $\mathrm{G}$ star varies between 10 and $20 \mathrm{MK}$ while those around the $\mathrm{K}$ star between 20 and 60 MK. This indicates that the corona of the secondary $\mathrm{K}$ giant is hotter than the corona of the G primary star. This possibility was also hinted in Walter et al. (1983).

In Fig. 2 we compare the images of AR Lac corona as obtained from $E X$ $O S A T, R O S A T$ and $A S C A$ observations. These images describe the states of AR Lac corona over 9 years. One observes quite a dramatic change in the overall distribution of the X-ray corona during this period. 
EXOSAT LE]T Ju] 84

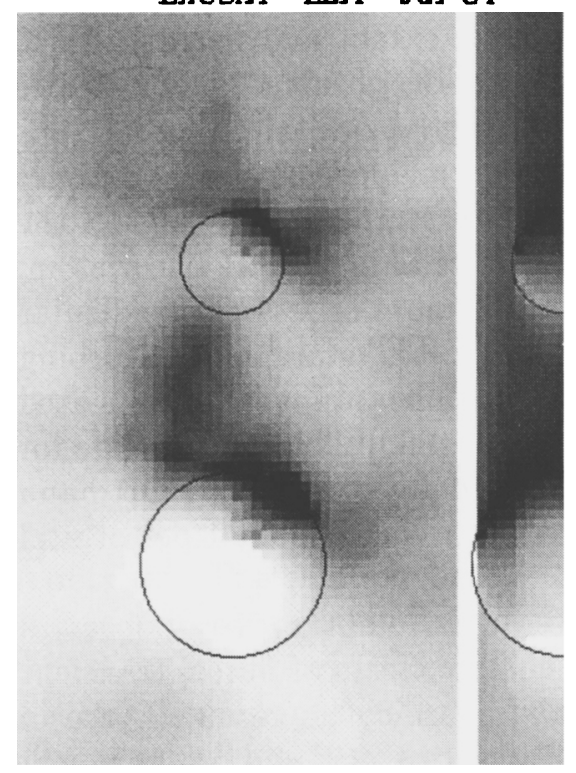

ASCA S]S Jun 93

\section{AR LAC}

ASCA GIS Jun 93

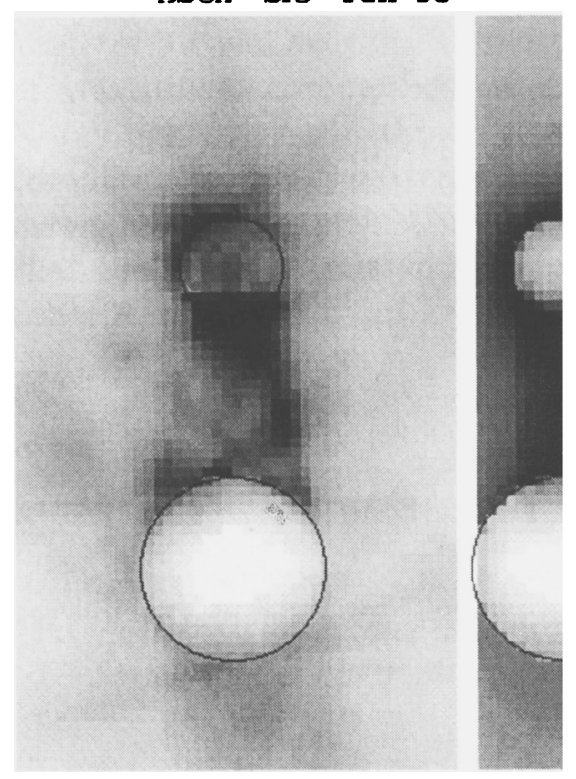

ROSAT PSPC DeC 90
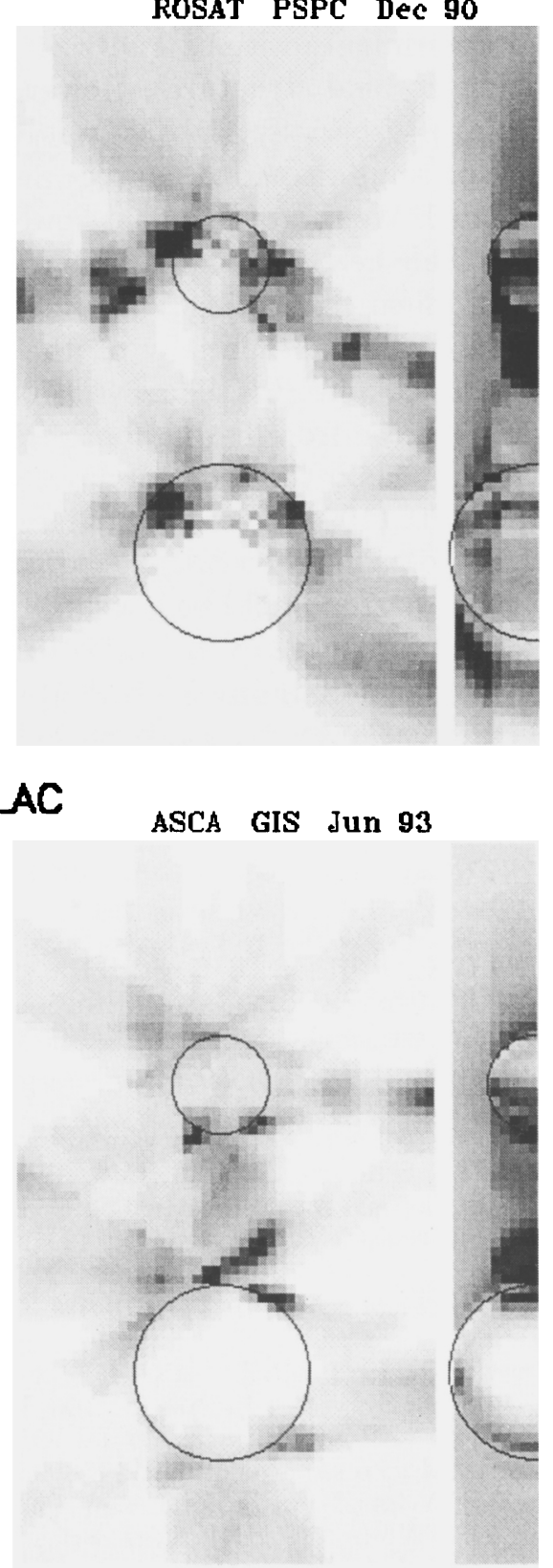

Figure 2. Comparison of the structure of AR lac X-ray corona as obtained from EXOSAT (a), ROSAT (b) and $A S C A$ SIS (c) and GIS (d) data analysis. For all panels top view and a side view for phase $0.25\left(90^{\circ}\right)$ are present.

\section{Conclusions}

Presented examples indicate that eclipse imaging of stellar coronae can provide valuable information about spatial distribution of hot coronal plasma 
around the stars. Modulation features present in the X-ray light curve allow us to determine positions and sizes (horizontal extent and height) of the emitting coronal structures. However the solution obtained is sometimes nonunique, especially for observations with partial orbital coverage. The reality of some extended sources and/or halo emission can be sometimes questioned. We must remember however, that for temperature $\sim 10 \mathrm{MK}$ the density scale height is comparable to the stellar radii in the AR Lac system. Magnetic field connecting both stars should be more or less perpendicular to stellar surfaces, especially on the inner surfaces of stars, not disturbing preassure scale heights. The extended halo component may be flare-heated plasma, ejected from flaring loops (White et al. 1990) The cooling time for this plasma is on the order of days, what exceedes, estimated by Ottmann and Schmitt (1990) frequency of large flares occurence (about 1 per orbital period).

The next step in the eclipse imaging studies is to obtain X-ray observations over multiple orbital cycles to exclude the effects of flares, transient brightness and to improve $\mathrm{S} / \mathrm{N}$ statistics and phase coverage. In all cases a joint observations programme including UV, optical and radio ranges will be highly desired.

\section{References}

Chambliss C.R., 1976, PASP 88, 762

Bedford D.K., Jeffries R.D., Geyer E.H., Vilhu O., 1990, MNRAS 243, 557

Culhane J.L., White N.E., Shafer R.A., Parmar A.N., 1990, MNRAS 243, 424

Guinan E.F., Carroll S.M., 1990, in Active Close Binaries, ed. C. İbanoğlu, Kluwer, Dordrecht, p. 7

Lemen J.R., Mewe R., Schrijver C.J., Fludra A., 1989, ApJ 341, 474

Ottmann R., Schmitt J.H.M.M., Kürster M. , 1993, ApJ 413, 710

Ottmann R., Schmitt J.H.M.M., 1994, A\&A 283, 871

Preś P., Siarkowski M., Sylwester J., 1995a, MNRAS 275, 43

Preś P., Siarkowski M., Sylwester J., 1995b, JOSO Annual Report 1994, ed. M. Saniga, p.199

Schmitt J.H.M.M., 1992, in Variabilities in Stars and Galaxies, ed. G. Klare, SpringerVerlag, p. 188

Siarkowski M., 1992, MNRAS 259, 453

Siarkowski M., Sylwester J., Preś P., 1994, in Solar Coronal Structure, Proc. IAU Colloq. 144, eds. V. Rusin, P.Heinzel and J.-C. Vial, VEDA, Bratislava, p. 207

Siarkowski M., Preś P., White N.E., Singh K.P., Drake S.A., 1995, ApJ, submitted

Singh K.P., White N.E., Drake S.A., 1995, ApJ, submitted

Swank J.H., White N.E., Holt S.S., Becker R.H., 1981, ApJ 246, 214

Vesecky J.F., Antiochos S.K., Underwood J.H., 1979, ApJ 233, 987

Walter F.M., Gibson D.M., Basri G.S., 1983, ApJ 267, 665

White N.E., Shafer R.A., Horne K., Parmar A.N., Culhane J.L., 1990, ApJ 350, 776

White N.E. et al. 1994, PASJ 46, L97 